\title{
LINEAR ISOTROPY GROUP OF AN AFFINE SYMMETRIC SPACE
}

\author{
JUN NAGASAWA
}

Abstract. Let $K$ be a subgroup of the general linear group $\operatorname{GL}(n)$. The author found a necessary and sufficient condition that there exist an $n$-dimensional simply connected affine symmetric space $M$ such that $K$ coincides with the linear isotropy group of all affine automorphisms of $M$ at some point in $M$.

Let $M$ be an $n$-dimensional manifold with affine connection, $A(M)$ the group of all affine automorphisms of $M, H_{p}$ the subgroup of $A(M)$ consisting of all elements of $A(M)$ which fix a point $p$ in $M$, and $d H_{p}$ the linear isotropy group determined by $H_{p}$. Let $V$ be an $n$-dimensional vector space, GL( $n)$ the general linear group of $V$, and $K$ a subgroup of $\mathrm{GL}(n)$. We shall find a necessary and sufficient condition that there exists a simply connected affine symmetric space $M$ such that $K$ coincides with the linear isotropy group $d H_{p}$ at some point $p$ in $M$. We discussed similar problems for a Riemannian symmetric space [6]. First of all we shall prove the following:

Lemma. Let $T$ be a tensor in $V \otimes V^{*} \otimes V^{*} \otimes V^{*}$ which satisfies the following conditions.

(1) $T_{\cdot j k l}^{i}=-T_{\cdot j l k}^{i}$,

(2) $T_{\cdot j k l}^{i}+T_{\cdot k l j}^{i}+T_{\cdot l j k}^{i}=0$,

(3) $T_{\cdot h m n}^{i} T_{\cdot j k l}^{h}-T_{\cdot{ }_{j m n}}^{h} T_{\cdot h k l}^{i}-T_{\cdot k m n}^{h} T_{\cdot{ }^{\prime} h l}^{i}-T_{\cdot l m n}^{h} T_{\cdot j k h}^{i}=0$, where $T_{. j k l}^{\prime}$ are the components of $T$. Then there is an affine symmetric space whose curvature tensor at some point of it coincides with $T$.

Proof. We integrate the following differential equations.

$$
\partial \bar{\omega}^{i} / \partial t=d a^{i}+a^{k} \bar{\omega}_{k}^{i}, \quad \partial \bar{\omega}_{k}^{i} / \partial t=T_{\cdot k j l}^{i} a^{j} \bar{\omega}^{l},
$$

with initial conditions $\left(\bar{\omega}^{i}\right)_{t=0}=0,\left(\bar{\omega}_{k}^{i}\right)_{t=0}=0$.

The solutions $\bar{\omega}^{i}, \bar{\omega}_{k}^{i}$ are linear forms in $d a^{1}, \cdots, d a^{n}$ whose coefficients are integral functions of $t, a^{1}, \cdots, a^{n}$. If we set $t=1$ and replace $a^{i}$ by $x^{i}$, we have forms $\omega^{i}(x, d x), \omega_{j}^{i}(x, d x)$. Since the determinant of the

Received by the editors June 16, 1971.

AMS 1970 subject classifications. Primary 53C35.

Key words and phrases. Linear isotropy group, affine symmetric space. 
coefficients of $d x^{1}, \cdots, d x^{n}$ in $\omega^{1}, \cdots, \omega^{n}$ is equal to 1 for $x^{1}=0, \cdots$, $x^{n}=0$, we find a positive number $\varepsilon$ such that this determinant is different from zero for $\left|x^{i}\right|<\varepsilon \quad(i=1,2, \cdots, n)$. Therefore we find an analytic manifold $M$ with analytic forms $\omega^{i}, \omega_{i}^{i}$ of which $\omega^{1}, \cdots, \omega^{n}$ are linearly independent. We shall show that the forms $\omega^{i}, \omega_{;}^{i}$ satisfy the structure equations

$$
d \omega^{i}=\omega^{k} \wedge \omega_{k}^{i}, \quad d \omega_{j}^{i}=\omega_{j}^{l} \wedge \omega_{l}^{i}+\frac{1}{2} T_{\cdot j k l}^{i} \omega^{k} \wedge \omega^{l} .
$$

It is sufficient to prove that these equations are satisfied by the forms $\bar{\omega}^{i}(t, a ; d a)$ and $\bar{\omega}_{j}^{i}(t, a ; d a)$ where $d \bar{\omega}^{i}, d \bar{\omega}_{j}^{i}$ are calculated regarding $t$ as a constant. If we pose

$$
d \bar{\omega}^{i}=\bar{\omega}^{k} \wedge \bar{\omega}_{k}^{i}+\varepsilon^{i}, \quad d \bar{\omega}_{j}^{i}=\bar{\omega}_{j}^{l} \wedge \bar{\omega}_{l}^{i}+\frac{1}{2} T_{\cdot j k l}^{i} \bar{\omega}^{k} \wedge \bar{\omega}^{l}+\varepsilon_{j}^{i},
$$

$\varepsilon^{i}, \varepsilon_{j}^{i}$ are quadratic differential forms in $d a^{1}, \cdots, d a^{n}$ and vanish for $t=0$. We shall prove the following equations

$$
\partial \varepsilon^{i} / \partial t=a^{k} \varepsilon_{k}^{i}, \quad \partial \varepsilon_{k}^{i} / \partial t=T_{\cdot k j l}^{i} a^{j} \varepsilon^{l},
$$

which show that $\varepsilon^{i}=0, \varepsilon_{k}^{i}=0$ for all $t \in R$.

From $d\left(\partial \bar{\omega}^{i} / \partial t\right)=(\partial / \partial t) d \bar{\omega}^{i}$, we find

$$
\partial \varepsilon^{i} / \partial t=a^{k} \varepsilon_{k}^{i}+\frac{1}{2} a^{k} \bar{\omega}^{j} \wedge \bar{\omega}^{i}\left(T_{\cdot k j h}^{i}-2 T_{\cdot j k h}^{i}\right) .
$$

But by (1) and (2) we get $T_{\cdot k j h}^{i}-2 T_{. j k h}^{i}=-2 T_{\cdot(j|k| h)}^{i}$. Therefore we have $\partial \varepsilon^{i} / \partial t=a^{k} \varepsilon_{k}^{i}$. From $d\left(\partial \bar{\omega}_{k}^{i} j \partial t\right)=(\partial / \partial t) d \bar{\omega}_{k}^{i}$ we find

$$
\partial \varepsilon_{k}^{i} / \partial t=T_{\cdot k j l}^{i} a^{j} \varepsilon^{l}+a^{j} \bar{\omega}^{h} \wedge\left(T_{\cdot l j h}^{i} \bar{\omega}_{k}^{l}+T_{\cdot k l h}^{i} \bar{\omega}_{j}^{l}+T_{\cdot k j l}^{i} \bar{\omega}_{h}^{l}-T_{\cdot k j h}^{l} \bar{\omega}_{l}^{i}\right)
$$

Consider the forms $\phi_{j k h}^{i}$ defined by

We get

$$
\phi_{j k h}^{i}=T_{\cdot l j h}^{i} \bar{\omega}_{k}^{i}+T_{\cdot k l h}^{i} \bar{\omega}_{j}^{l}+T_{\cdot k j l}^{i} \bar{\omega}_{h}^{l}-T_{\cdot k j h}^{l} \bar{\omega}_{l}^{i}
$$

$$
\partial \phi_{j k h}^{i} / \partial t=a^{m} \bar{\omega}^{n}\left(T_{\cdot l j h}^{i} T_{\cdot k m n}^{l}+T_{\cdot k l h}^{i} T_{\cdot j m n}^{l}+T_{\cdot k j l}^{i} T_{\cdot h m n}^{l}-T_{\cdot k j h}^{l} T_{\cdot l m n}^{i}\right) .
$$

By (3) we have $\partial \phi_{j k h}^{i} / \partial t=0$. Since $\phi_{j k h}^{i}=0$ for $t=0$, we have $\phi_{j k h}^{i}=0$ for all $t \in R$. Then we have $\partial \varepsilon_{k}^{i} / \partial t=T_{. k j l}^{i} a^{j} \varepsilon^{l}$. Therefore $M$ is an affinely connected manifold of class $c^{\omega}$. Consider the point $o=(0, \cdots, 0)$ in $M$. If we identify the tangent space $M_{o}$ with $V$, the curvature tensor at $o$ coincides with $T$. Since $\phi_{j k h}^{i}=0$ means that the covariant differential of the curvature tensor vanishes, $M$ is an affine locally symmetric space. Then an open neighborhood of $o$ can be extended to an affine symmetric space [2, p. 58].

REMARK. E. Cartan proved a similar theorem for Riemannian symmetric spaces $[1$, p. 265]. 
For an element $A$ of $\mathrm{GL}(n)$ we denote by $\tilde{A}$ the Kronecker product $A \otimes A^{*} \otimes A^{*} \otimes A^{*}$, where $A^{*}$ is the dual of $A$.

THEOREM. Let $K$ be a subgroup of $\mathrm{GL}(n)$. In order that there exists a simply connected affine symmetric space $M$ such that $K$ coincides with the linear isotropy group $d H_{p}$ at some point $p$ in $M$, it is necessary and sufficient that there exists a tensor $T$ in $V \otimes V^{*} \otimes V^{*} \otimes V^{*}$ such that the following conditions are satisfied.

(1) $T_{\cdot i k l}^{i}=-T_{\cdot j l k}^{i}$,

(2) $T_{\cdot j k l}^{i}+T_{\cdot k l j}^{i}+T_{\cdot l j k}^{i}=0$,

(3) $T_{\cdot h m n}^{i} T_{\cdot j k l}^{h}-T_{\cdot{ }_{j m n}}^{h} T_{\cdot h k l}^{i}-T_{\cdot k m n}^{h} T_{\cdot j h l}^{i}-T_{\cdot l m n}^{h} T_{\cdot{ }_{j k h}}^{i}=0$,

(4) $K=\{A \in \mathrm{GL}(n) ; \tilde{A} T=T\}$, where $T_{\cdot j k l}^{i}$ are the components of $T$.

Proof. Let $M$ be a simply connected affine symmetric space whose linear isotropy group at $p(\in M)$ is $K$. Since $M$ is locally symmetric, from the Ricci identity we have

$$
\begin{aligned}
0 & =\nabla_{n} \nabla_{m} R_{\cdot j k l}^{i}-\nabla_{m} \nabla_{n} R_{\cdot j k l}^{i} \\
& =R_{\cdot h m n}^{i} R_{\cdot j k l}^{h}-R_{\cdot j m n}^{h} R_{\cdot h k l}^{i}-R_{\cdot k m n}^{h} R_{\cdot j h l}^{i}-R_{\cdot l m n}^{h} R_{\cdot j k h}^{i},
\end{aligned}
$$

where $R_{. j k l}^{i}$ are the components of the curvature tensor $R$. Since $M$ is a simply connected, complete affine locally symmetric space, we have $d H_{p}=\left\{A \in \mathrm{GL}(n) ; \tilde{A} R_{p}=R_{p}\right\}$ [5]. If we set $T=R_{p}$, then $T$ satisfies (1)-(4).

Conversely if a tensor $T$ in $V \otimes V^{*} \otimes V^{*} \otimes V^{*}$ satisfies (1)-(4), by the above lemma we find an affine symmetric space $M$ and a point $p$ in $M$ at which $R_{p}=T$. Considering the universal covering manifold of $M$ if necessary, $M$ may be assumed to be simply connected. Then we have $d H_{p}=K$.

Let $K$ be a subgroup of $\mathrm{GL}(n)$ which satisfies the conditions of the above theorem. We denote by $\mathfrak{T}_{k}$ the set of all tensors in $V \otimes V^{*} \otimes V^{*} \otimes V^{*}$ which satisfy together with $K$ the above conditions and by $\mathfrak{G}_{k}$ the set of all simply connected affine symmetric spaces with linear isotropy group $K$. If $M$ and $M^{\prime}$ are affinely isomorphic spaces in $\mathfrak{G}_{k}$, we identify $M$ with $M^{\prime}$. Let $T$ and $T^{\prime}$ be tensors in $\mathfrak{I}_{k}$. They are said to be equivalent if there is $A$ in $\mathrm{GL}(n)$ such that $T^{\prime}=\tilde{A} T$. We denote by $\widetilde{I}_{k / \sim}$ the equivalent classes of $\mathfrak{I}_{k}$.

COROLlaRY. There is a one-to-one correspondence between $\mathfrak{I}_{k / \sim}$ and $\mathfrak{G}_{\boldsymbol{k}}$.

Proof. Let. $T$ be a tensor in $\mathfrak{I}_{k}$. Then by above theorem there is a space $M$ in $\mathfrak{G}_{k}$ and a point $p$ in $M$ such that $d H_{p}=K$ and $R_{p}=T$. Let $M^{\prime}$ be a space in $\mathfrak{G}_{k}$ and a point $p^{\prime}$ in $M^{\prime}$ such that $d H_{p^{\prime}}^{\prime}=K$ and $R_{p^{\prime}}^{\prime}=T$. If we identify the tangent space $M_{p}$ with the tangent space $M_{p}^{\prime}$, by a transformation $I, I$ maps $R_{p}$ in $R_{p^{\prime}}^{\prime}$. Since $M$ and $M^{\prime}$ are simply connected, $I$ 
induces an affine isomorphism of $M$ onto $M^{\prime}[3, \mathrm{p}$. 265]. Therefore a mapping $\lambda: \mathfrak{I}_{k} \rightarrow \mathfrak{G}_{k}$ is defined. We see by the above theorem that $\lambda$ is surjective. Let $T$ and $T^{\prime}$ be tensors in $\widetilde{I}_{k}$ such that $\lambda(T)=\lambda\left(T^{\prime}\right)$. Then there are a space $M$ in $\mathfrak{G}_{k}$ and points $p$ and $p^{\prime}$ in $M$ such that $R_{p}=T$, $R_{p^{\prime}}=T$. Since there is an affine automorphism $\varphi$ of $M$ such that $\varphi(p)=p^{\prime}$ [4, p. 223], by identifying $M_{p}$ with $M_{p^{\prime}}$ we have $(d \varphi)_{p} \in \mathrm{GL}(n)$ and $R_{p^{\prime}}=$ $\left((d \varphi)^{\sim}\right)_{p} R_{p}$. Therefore $T$ and $T^{\prime}$ are equivalent. Conversely if $T$ and $T^{\prime}$ are equivalent in $\mathfrak{I}_{k}$, clearly we have $\lambda(T)=\lambda\left(T^{\prime}\right)$.

\section{REFERENCES}

1. É. Cartan, Leçons sur la géométrie des espaces de Riemann, 2nd. ed., GauthierVillars, Paris, 1946. MR 8, 602.

2. K. Nomizu, Invariant affine connections on homogeneous spaces, Amer. J. Math. 76 (1954), 33-65. MR 15, 468.

3. S. Kobayashi and K. Nomizu, Foundations of differential geometry. Vol. 1, Interscience, New York, 1963. MR 27 \#2945.

4. - Foundations of differential geometry. Vol. 2, Interscience Tracts in Pure and Appl. Math., no. 15, Interscience, New York, 1969. MR 38 \#6501.

5. J. Nagasawa, On infinitesimal linear isotropy group of an affinely connected manifold, Proc. Japan Acad. 41 (1965), 553-557. MR 34 \#740.

6. - Linear isotropy group of a symmetric space, Mem. Fac. Ed. Kumamoto Univ. Sect. 117 (1969), 1-2. MR 40 \#5792.

Faculty of Education, Kumamoto University, Kumamoto 860, Japan 